\title{
Un Sistema Didáctico para la Enseñanza - Aprendizaje de la Física
}

\author{
(A Didactic System for the Physics Teaching - Learning) \\ José Ricardo Campelo Arruda \\ Instituto de Física, Universidade do Estado do Rio de Janeiro \\ Av. São Francisco Xavier 524, Rio de Janeiro, RJ, Brasil, CEP 20550-013 \\ Email:arruda@uerj.br \\ José Marín Antuña \\ Facultad de Física, Universidad de La Habana \\ San Lazaro y L, Havana, 10400 Cuba \\ Email: marin@ff.oc.uh.cu
}

Recebido em 06 de Julho de 2001. Aceito em 17 de Agosto 2001.

El objetivo de esta investigación es saber cuales serán las regularidades formativas de las estructuras lógicas y psicológicas básicas del pensamiento teórico en los estudiantes en los cursos del área tecnológica, como consecuencia de la enseñanza del modelo didáctico propuesto, que utiliza los fundamentos de la Teoría de la Actividad, Generalización Teórica y el Método Sistémico Estructuralfuncional para la organización del contenido a ser desarrollado. La investigación realizada demuestra que es posible elevar el nivel de desarrollo del pensamiento lógico a través de la enseñanza de una Asignatura, como la Física, cuando utiliza la Actividad de Estudio y el método estructural-funcional de organización del contenido. Los resultados de la investigación apuntan que el modelo didáctico propuesto, pone en correspondencia el método científico de obtener conocimiento descubierto por la Epistemología de la Física, con la actividad de enseñanza y psicología del aprendizaje de esta ciencia. La investigación mostró que la enseñanza tradicional que prioriza la dicotomía entre la enseñanza de la parte teórica y experimental, no favorece un aprendizaje efectivo; por consiguiente, es necesario considerar el proceso enseñanza-aprendizaje de la Física de forma sistémica y desarrollado como un conjunto de partes interligadas y que posee una lógica interna característica, donde la evaluación desempeña importante papel en la realimentación del proceso, necesario a conseguir los objetivos planeados, tanto en la visión del profesor, como en la visión del estudiante, proporcionando una enseñanza de calidad.

The objective of this investigation is to know which will be the formative regularities of the basic logical and psychological structures of the theoretical thought in the students in the courses of the technological area, as a consequence of the teaching of the proposed didactic model, that uses the foundations of the Theory of the Activity, Theoretical Generalization and the Sistemic Structural-Functional Method for organization of the content to be developed. The accomplished investigation demonstrates that it is possible to elevate the level of development of the logical through the teaching of a Discipline, as the Physics, when it uses the Activity of Study and the structural-functional method of organization of the content. The results of the investigation point that the proposed didactic model, puts in correspondence the scientific method of obtaining of the knowledge discovered by the Physics Epistemology, with the teaching activity and the psychology of the learning of this science. The investigation showed that the traditional teaching that it prioritizes the dichotomy among the teaching of the theoretical and experimental part, doesn't favor an effective learning; therefore, it is necessary to consider the Physics process teaching-learning in systemic form and developed as a group of interlinked parts, possessing a logic interns characteristic, where the evaluation plays important paper in the feedback of the process, necessary to get the planned objectives, so much in the teacher's vision, as in the student's vision, providing a quality teaching. 


\section{Introducción}

La educación es un fenómeno universal necesario para la continuidad cultural de la humanidad a través de la cual las generaciones antecedentes preparan a las siguientes, en un continuo de transferencia de informaciones, conocimientos y habilidades.

De esa manera, cada nueva generación recibe los conocimientos sobre el mundo sólo a través del contacto con este, porque ellos son transmitidos por la generación antecedente, por medio de los objetos y a través de una organización especial de la actividad de la nueva generación con estos objetos.

Por consiguiente, el efecto de la enseñanza y el desarrollo es resultado directo de la actividad de los estudiantes que los une al mundo circundante.

De esto se concluye que las generaciones antecedentes transmiten a las siguientes no sólo el conjunto de los objetos sociales materiales y las ideas, sino también, juntamente con ellos, el sistema de los métodos socialmente elaborados de las acciones.

De esa manera, las demandas sociales en cierto momento histórico-social determinan los objetivos de la educación y estos se caracterizan en los contenidos y están estrechamente unidos a los métodos a utilizar en la enseñanza. Así, las Instituciones de Enseñanza y la sociedad son indivisibles," la sociedad vive y se desarrolla tal como aprende y aprende tal como quiere vivir" [Davidov, 1991, pg 118].

Para entender el problema de la enseñanza de la Física, es necesario buscar la causa y el efecto de la situación-problema en el proceso de enseñanzaaprendizaje de esta ciencia. Esta problemática del proceso interrelaciona los aspectos siguientes: epistemológico, psicológico y didáctico.

Estes factores parecen reforzar la idea de que la Física enseñada en la Universidad no parece ejercer influencia significativa en la formación cultural y científico - técnica del estudiante ni está contribuyendo satisfactoriamente al aprendizaje de conceptos y leyes, para la habilidad de operar en su especialidad, o para el perfeccionamiento del razonamiento en la solución de problemas de Física o de la vida diaria.

"La falta de motivación de los estudiantes en el aprendizaje de Física, tiene sobre el punto central el conflicto de objetivos; por un lado los Profesores con su formalismo matemático desprovisto de significado, no consiguen modificar en los estudiantes las estructuras anteriores de los conceptos adquiridas en otra fase de su formación intelectual; por otro lado el estudiante con sus objetivos y motivación de su especialización no consigue asimilar los conceptos físicos y entender la función de la enseñanza de Física para su especialización" [Arruda, Marín, Armas 1999, pg 76].

Dentro del problema del proceso enseñanzaaprendizaje de la Física, aparece la necesidad de un nuevo paradigma para la enseñanza de Física, apoy- ado en la metodología científica y que explicite el interdisciplinaridade, el valor social y implicaciones que tiene la enseñanza y la ciencia Física para la sociedad. "Los procedimientos y aplicaciones del tal paradigma son tan necesarios a la ciencia como las leyes y teorías paradigmáticas - y ellos tienen los mismos efectos" [Kuhn 1998, pg 87].

Estamos de acuerdo con [Alvarez 1996, pg 22], al afirmar que "en las universidades, la ciencia tiene una intención profesional, la profesión se apoya en las ciencias y en ambos casos la esencia está en encontrar los métodos que posibiliten formar profesionales con un profundo dominio de la lógica particular de cada ciencia y de su metodología general de investigación”.

\section{Objetivos del estudio}

Específicamente, respecto a la ciencia Física, ésta es una ciencia que necesita particularmente del razonamiento deductivo para su comprensión y acostumbradamente se ve como una asignatura de asimilación difícil por parte de los estudiantes del área tecnológica. Lo que se desea comprobar es que gran parte de esta dificultad no está en la complejidad de la ciencia en símisma, sino en el hecho de que los estudiantes no desarrollaron apropiadamente la formación de los procedimientos lógicos del pensamiento, así como, la enseñanza de la Física no haberse desarrollado científicamente con métodos elaborados, capaces de formar tales procedimientos.

Los objetivos del presente trabajo son: a) elaborar un Sistema Didáctico para la enseñanza de la Física, en correspondencia con los fundamentos de la Teoría de la Actividad; usando aspectos de la Generalización Teórica y el método sistémico estructuralfuncional para la organización del contenido a ser desarrollado; b) investigar cuales serán las regularidades formativas de las estructuras lógicas y psicológicas básicas del pensamiento teórico en los estudiantes de los cursos del área tecnológica, como consecuencia de la enseñanza de invariantes de un tema de la ciencia Física; c) hacer una experimentación del modelo didáctico propuesto en el curso de Física con el contenido de Mecánica Clásica para el área tecnológica; ch) proponer recomendaciones a partir de los resultados obtenidos en la experimentación realizada de manera a contribuir para la efectividad del proceso enseñanza-aprendizaje de la ciencia Física.

\section{Fundamentación teórica}

El trabajo presenta una propuesta de un sistema didáctico para la enseñanza-aprendizaje de la Física con los fundamentos teóricos del enfoque histórico-cultural, cujos princípios son esenciales para el logro de una enseñanza científica y desarrolladora. Las ideias de Vygotsky situán el origen de los proceso psicológicos supe- 
riores en el plano interpsicológico, en el que se forma el plano intrapsicológico, muy importante en su teoria es el concepto de zona de desarrollo próximo importante para el enfoque de este investigación.

La fundamentación teórica metodológica de nuestra investigación se sustenta en los aportes de la escuela histórico cultural surgida con Vygotsky y se destacan en esta concepción el caracter activo y socio histórico del desarrollo psiquico, asi como la unidade entre enseñanza y desarrollo, en este sentido se destacan las investigaciones de P. Ya. Galperin [1982], N.F. Talizina [1987, 1988], Davidov [1974], Leontiev[1983], Fariñas[1995], Salmina[1989], entre otros.

El pensamiento lógico constituye un nivel superior en la actividad cognoscitiva del hombre que da posibilidad de razonar de manera consciente cada situación y llegar a conocer la esencia de los objetos y fenomenos a partir de los cuales puede establecer nuevos conocimientos o nuevas relaciones entre los elementos ya conocidos.

Para Vygotsky - el concepto espontáneo, cotidiano, habitualmente aparece en el niño, después del contacto de este con objetos o fenómenos. Es sólo mucho más tarde que él será consciente de la noción del objeto y aprenderá a hacer operaciones abstractas con el objeto. Inversamente, el concepto científico aparece con el establecimiento de una conexión indirecta con el objeto: primero el niño se confronta con una noción e aprende a establecer relaciones lógicas entre las nociones; sólo después de eso tendrá conciencia del objeto. Es como si el concepto científico hiciera un camino hasta el objeto, a través de la experiencia del niño en el decurrir del desarrollo de la experiencia.

Podemos decir que el concepto espontáneo es desarrollado por un proceso inductivo - de las propiedades elementales e inferiores a las propiedades superiores, mientras que el concepto científico es desarrollado por un proceso deductivo - de las propiedades complejas y superiores a las propiedades elementales e inferiores.

Los conceptos científicos bajan a través de los conceptos cotidianos, mientras los últimos suben a través de los primeros ya mencionados. Sin embargo, afirma Vygotsky, aunque opuestos, esos dos procesos se encuentran íntimamente ligados. En este contexto, y bajo la teoría de la actividad, el proceso de elaboración de los conceptos científicos y teóricos debe organizarse según un esquema deductivo - de lo abstracto hacia lo concreto. El conocimiento teórico es proveniente de una transformación de los objetos y refleja las relaciones entre sus propiedades y sus conexiones interiores. En el momento en que el pensamiento reproduce un objeto bajo la forma de conocimiento teórico, supera las representaciones sensoriales; la materialización exige la transformación del conocimiento en una teoría desarrollada a través de una deducción, e una explicación de las manifestaciones concretas del sistema, a partir de la base fundamental.

La fijación de los resultados se expresa, al princi- pio, por los diferentes modos de la actividad intelectual, e, en un segundo momento, por diferentes sistemas semióticos. Es esta la visión para entender un fenómeno físico, es decir, expresarlo en forma de concepto.

Expresar el objeto en forma de concepto, significa entender su esencia. No podemos imaginar un movimiento en caída libre de un cuerpo, sin esbozarlo mentalmente. Aun así, el esbozo, la concepción mental, es solamente la reproducción, la construcción del objeto en el plano ideal.

Los símbolos y las señales son los medios de construcción de la objetividad idealizada. La forma sensorial del símbolo es similar a los objetos que representa. Los símbolos son representantes sensoriales, de una cierta manera, de objetos; en la Física, la modelación es un tipo peculiar de idealización simbólicosemiótica.

Los modelos semióticos requieren una interpretación especial, sin la cual pierden la función de modelos. Por ejemplo, la fórmula física $\vec{F}=\vec{m} a$ es un modelo semiótico; la relación y la sucesión de cuyos elementos transmiten el carácter de la relación física real, la estructura de la fuerza.

Toda la relación entre las generalizaciones simbólicas - la fórmula física - refleja las relaciones correspondientes entre las cosas reales de la naturaleza. Los modelos semióticos reflejan las conexiones y relaciones de los objetos reales de la naturaleza e en este sentido, es posible considerar las conexiones y relaciones entre las señales físicas como expresión visual del original.

En síntesis, el pensamiento teórico se descompone en varios elementos que son:

a) La reflexión que consiste en el descubrimiento por parte del sujeto, de las razones de sus acciones y de su correspondencia con las condiciones del problema;

b) El análisis del contenido del problema, que busca el principio o el modo universal para su resolución, para transferirlo para una clase entera de problemas similares;

c) El plan interior de las acciones que aseguran su planificación y su efectivación.

En una clase de Física, y en ocasión de la elaboración de los conceptos físicos, es necesario analizar y definir las relaciones existentes entre las particularidades del funcionamiento de los conceptos y de los conocimientos de los estudiantes, en el plano de la conciencia social e individual, es decir, no podemos ignorar el sujeto y sus ideas. Esta enseñanza debe orientarse al desarrollo y al perfeccionamiento de la conciencia y de la personalidad de los estudiantes.

La elaboración del Sistema Didáctico propuesto presupone el diseño de un nuevo contenido de la Física, bien como la modelación y experimentación del objeto físico a transformar, basado en la actividad de estudio.

Esto es posible cuando los estudiantes efetúan aquellas transformaciones específicas de los objetos físicos, 
en su propria práctica laboral se modelan y recrean las propriedades internas del objeto que se converten en contenido del concepto físico.

Estas acciones que revelan y constryuen las conexiones esenciales y generales de los objetos físicos, sirven de fuente para las abstraciones, generalizaciones y conceptos teóricos.

De acuerdo con Davidov [Davidov, 1991, pg 131], los conocimientos, adquiridos en el proceso de la actividad y en la forma de auténticos conceptos teóricos, reflejan por esencia, las propriedades internas de los objetos y aseguran que el individuo se oriente por ellos en la resolución de tareas prácticas.

El contenido de las asignaturas de Física debe favorecer la formación, en los estudiantes, del pensamiento teórico que se forma durante la realización de la actividad de estudio. Por eso es indispensable elaborar el contenido de las disciplinas de Física en correspondencia com las particularidades y la estrutura de la actividad de estudio de Física.

De acuerdo a la teoria de formación por etapas de las acciones mentales, el movimiento individual puede ser garantizado por la estruturación especial de la asignatura, cuando en el mismo comienzo de la enseñanza de la Física, se exponen los conceptos físicos más generales que forman la asignatura de Física, en acuerdo com tercer tipo de aprendizaje según Galperin.

Dentro del tercer tipo de aprendizaje, dirigido a la formación de los conocimientos teóricos, es posible estruturar la asignatura de Física.

La estruturación del programa de la asignatura Física, comenza de la posición de que con la lógica de estruturación, el programa de Física debe formar determinado tipo-sistémico de orientación en los fenómenos físicos de la realidad estudiada.

El pensamiento teórico en la etapa actual es la forma específica de representación de la realidad : éste refleja la cosa como sistema, su invariante (estrutura), las posibles variantes de su existencia y las leys (limites de estabilidad), el orígen de las propriedades esenciales del sistema com su estrutura interna [Salmina, 1989, pg 7].

La organización del contenido de la Física basado en el método sistémico estructural- funcional, destaca el esquema completo del objeto físico de estudio, construido en la lógica del análisis sistémico y que reproduce el quadro completo de la descrición teórica del objeto físico estudiado.

Sobre la base de la fundamentación arriba realizada, formularemos los princípios del Sistema Didáctico propuesto.

\section{Sistema didáctico propuesto}

La sociedad a partir del estado de su desarrollo y de sus aspiraciones configura las características que deben poseer los hombres que aspira a formar.
En esa perspectiva, el proceso educativo tiene que ser analizado en una visión sistémica, establecer las ideas básicas y las leyes que se derivan de las relaciones del sistema con el medio - la sociedad e internamente al sistema.

En ese sentido, los objetivos de la enseñanza son la expresión pedagógica de las demandas que la sociedad necesita para formar un profesional que responda a los progresos científico-técnicos de la actualidad.

Existen tres eslabones importantes en el proceso de enseñanza: los OBJETIVOS que constituyen el núcleo central y responden a la pregunta: para que enseñamos?, el CONTENIDO, que responde a la pregunta: qué enseñamos?, y el tercero, que se refiere al MONTAJE DEL PROCESO DE ENSEÑANZAAPRENDIZAJE, sería: cómo hay que enseñar para alcanzar los objetivos planteados?

Los objetivos tienen tres niveles, el primero, general para todos los países del mundo, tiene que ver con las particularidades de la época en que vivimos. El segundo nivel de objetivos se refiere al cumplimiento de los requisitos planteados por el régimen sociopolítico del país. El tercer nivel de objetivos es muy propio, específico de cada especialidad; hablamos del perfil o modelo del especialista que se desea formar. [Talizina, 1997, pg 1].

El contenido es el resultado del conocimiento de la cultura universal o de la ciencia Física, necesario para asimilar y garantizar el cumplimiento de los objetivos propuestos.

El contenido presenta dos componentes esenciales para cumplimiento de su objetivo:

a) sistema de conocimientos; $y$

b) sistema de habilidades.

El conocimiento es el reflejo del objeto en la conciencia del hombre e incluye el conocimiento de habilidades y normas de conducta.

La habilidad es la acción que consiste en una serie de operaciones que tienen un objetivo general. En el proceso de enseñanza-aprendizaje de la Física, la habilidad caracteriza las acciones que el sujeto logra realizar al interactuar con su objeto de estudio, es decir, el objeto físico.

El contenido de la enseñanza de la Física en la práctica se reduce al documento que regula el plan de estudio y los programas de la Asignatura Física.

Cuando el contenido de la Asignatura Física se expone en un programa siempre se sigue la lógica de la ciencia Física, lo que normalmente se llama el conocimiento de la Asignatura.

La montaje del proceso enseñanza-aprendizaje y la correlación entre la ciencia Física y la Asignatura Física es uno de los aspectos más importantes en cuanto al contenido de la formación que recibe un estudiante del curso de Física, o especialista de los cursos del área tecnológica.

Hasta el momento estos aspectos se han explorado muy poco debido a la falta de un análisis más profundo 
para saber el papel que desempeña en la didáctica la estructura lógica del conocimiento contenido en las Asignaturas de Física que son impartidas para un curso en las Universidades o en las escuelas de enseñanza media.

En esa visión, existen dos puntos a considerar, es decir, la relación entre los elementos material y formal en la teoría del contenido de enseñanza de la Física y la teoría de enseñanza en la educación superior o media.

Es importante destacar que estos puntos no son excluyentes, pues el razonamiento no está reducido sólo a la adquisición de los conocimientos, esto incluye también las acciones mentales encaminadas para transformarlos.

El aprendizaje, junto al proceso de asimilación, debe funcionar también en todo momento con el proceso orientado a la constitución de un nuevo conocimiento que no era parte de la experiencia anterior del estudiante antes de ejecutar esta experiencia inmediata en la actividad cognoscitiva.

Sin embargo, la actividad constructiva de los estudiantes tiene su origen en el punto en que este proceso entra en interacción específica con los elementos de la actividad de estudio, es decir, con los conocimientos de los objetos y fenómenos del mundo externo como medios de la cognición.

Estas interacciones, así como los elementos integrantes de la actividad cognoscitiva, que ejecutan funciones diferentes en la actividad constructiva del estudiante, integran el contenido de su interés cognoscitivo.

Al comenzar el estudio de una Asignatura de Física, los estudiantes deben saber su composición y estructura lógica, así como, sus conexiones y relaciones estructurales.

La estructuración adecuada del material de estudio que atiende la teoría de la ciencia Física y sus elementos permite resolver una serie de tareas pertinentes al proceso de enseñanza- aprendizaje, como son:

a) Formar en los estudiantes durante la actividad de estudio un pensamiento teórico, es decir, la habilidad de tratar las teorías y sus elementos esenciales en sus conexiones y relaciones;

b) Ayudar a los estudiantes a asimilar el material de estudio de una manera lo más racional y efectiva posible, es decir, dominar los conceptos y las teorías en forma generalizada, tanto mediante el fundamento que permite desarrollarlas independientemente, como por medio del fundamento que permite concretizarlas;

c) Formar en los estudiantes criterios, habilidades y hábitos para utilizar individualmente los procedimientos cognoscitivos creativos.

Los principios fundamentales del Sistema Didáctico propuesto son:

a) Concepción sistémica estructural-funcional de organización de las asignaturas de la Física;

b) Enfoque fenomenológico de los conceptos físicos;

c) Realizar la enseñanza basada en el método científico, utilizando la actividad de estudio y orien- tada hacia la formación del pensamiento teórico de los estudiantes;

ch) Considerar el desarrollo, el aprendizaje y la enseñanza como elementos relacionados entre sí.

En este contexto, la enseñanza de la Física debe desarrollarse a través del tránsito de lo general a lo particular, resumiendo el núcleo que sirve como base a cada caso particular, para ver se todos estos casos son variantes del mismo objeto de estudio. El objeto de estudio viene dado como un conjunto de invariantes e incorpora un componente formativo que debe ser parte de una Asignatura y que está integrado por los conocimientos y las habilidades específicas.

Como punto fundamental, consideramos que los conceptos físicos que constituyen la Asignatura Física, considerada en símisma, deben ser adquiridos por los estudiantes por medio del análisis de las fuentes materiales - los objetos concretos, objetos en razón de los cuales esos mismos conceptos se tornan indispensables al aprendizaje.

De hecho, la forma de adquisición del conocimiento físico (y, por consiguiente, así debería ser su enseñanza), es un camino inductivo-deductivo. Específicamente: comenzando por el experimento, es decir, la base fenomenológica, se induce la ley general. Aún así, en un segundo momento, se pasa a la deducción; es decir, comenzando por las presuposiciones teóricas inducidas, se va de lo general a lo particular por medio de un desarrollo teórico-matemático, para obtener como consecuencia de esa teoría, el resultado que experimentalmente en un principio llevó a la inducción.

Así, primero se induce, poco después se deduce y se cierra el ciclo llegando al mismo punto de partida, pero, en un nivel alto y profundo de comprensión del fenómeno, en un nuevo giro de la espiral del conocimiento del objeto físico que se está estudiando.

Para la comprensión de un concepto físico, es necesario conocer su estructura y su funcionamiento que se traduce por la actualización de las relaciones recíprocas existentes entre los tres elementos: el modelo Físico, el plan fenomenológico de la descripción de la realidad y el aparato matemático formal de la teoría.

La asimilación de los conceptos que constituyen la Asignatura Física se da con base en el análisis de las condiciones de su origen, es decir, los conceptos no se dan como conocimientos listos.

De acuerdo con la teoría de la actividad del aprendizaje, concebida por Davidov, el objetivo de la actividad del aprendizaje es la adquisición, por parte de los estudiantes, tanto del contenido teórico y científico de los conceptos esenciales de un tema de la ciencia Física, como a partir de la lógica de su génesis. Ese contenido está ligado a los modos generalizados de acción, típicos de la ciencia Física, en el momento de su aprendizaje; los conceptos sirven como procedimientos de creación y de realización de la acción y servirán como base futura. 
Es necesario considerar el desarrollo, el aprendizaje y la enseñanza de la Física, como tres elementos relacionadas entre sí, de manera que el nivel de desarrollo efectivo condiciona los posibles aprendizajes que el estudiante puede realizar gracias a la enseñanza, pero esta, a su vez, puede modificar el nivel del desarrollo efectivo del estudiante, por medio del aprendizaje que promueve.

\section{La construcción del contenido en el enfoque sistémico estructural-funcional}

Analizando los libros texto de Física adoptados en Brasil para los cursos del área tecnológica, que denominamos convencionalmente de tradicional, lo es característico lo siguiente: la sucesión de la presentación de las partes del curso no se muestra al estudiante como un único sistema en interrelación con los temas que lo componen, es decir, que la lógica del curso se quiebra sin revelar y sin fundamentar su dinámica interna.

En muchos casos, algunos temas del curso aparecen generalmente como partes aisladas no relacionadas con otras. Con esto se explica la gran dificultad de una secuencia en la exposición del material en los cursos impartidos, así como en su integración con los contenidos y/o experiencias de laboratorio. Además, para los cursos existentes es característica la fragmentación de la exposición y el principio histórico de la exposición dentro de los distintos temas.

La tarea fundamental de la enseñanza a través de la generalización teórica en todos sus niveles no es proporcionar al estudiante una cierta cantidad de conocimiento asimilado, sino, principalmente, la formación del pensamiento teórico, lo que da a los estudiantes la posibilidad de guiarse individualmente en la información científica.

De acuerdo con [Salmina 1989, pg 1], "Las investigaciones psicológicas demuestran que el desarrollo de uno u otro nivel de formación del conocimiento y el desarrollo del pensamiento se determinan por el contenido del conocimiento asimilado".

La estructuración de una asignatura según la formación del conocimiento teórico puede ser la forma sistémica estructural - funcional, fundamentada bajo el tercer tipo de Base Orientadora de la Actividad del aprendizaje, dirigido a la formación de los conocimientos teóricos y de acuerdo con la teoría de las etapas de formación de acciones mentales de Galperin, que garantiza que el movimiento individual puede ser realizado por la estructuración especial de la asignatura, cuando en el principio de la enseñanza, se exponen los conocimientos más generales que forman la asignatura.

En este contexto, el objeto de estudio se presenta como un objeto complejo y tiene como objetivo la revelación del funcionamiento y desarrollo del objeto en sus características internas y externas.

De esa manera, la tarea principal es la investigación del esquema del principio de desmembración del objeto, lo que en la conservación del todo, garantizará la posibilidad de su análisis.

El aspecto principal de la estructuración de la asignatura de la Física a través del modelo sistémico estructural-funcional, es lo que permite describir el objeto complejo en su estado conservado, como si fuera en sincronía, resaltando en él la composición y estructura que garantizan su excelente funcionamiento, en un sistema mayor o la existencia estable en un medio circundante.

En la construcción de las asignaturas de Física que reflejan este tipo de investigaciones sistémicas, las características estables estructural-funcional de cada nivel del sistema se denominan invariantes del sistema. En este modelo el objeto se estudia como una serie de sub- sistemas estructural-funcionales jerarquizados y de niveles con la variante de cada nivel.

El procedimiento para construir una asignatura en correspondencia con los principios del enfoque sistémico estructural-funcional de un programa de estudios de una asignatura de la Física, tiene que partir de la posición de que con la lógica de la estructuración, el programa debe formar cierto tipo (sistémico) de orientación en los fenómenos de la realidad estudiada.

\section{Actividad de estudio de física}

La Actividad de Estudio de Física es la base metodológica en que se desarrolla el proceso de enseñanza-aprendizaje de la Física, siendo un conjunto de acciones y operaciones mentales o prácticas a ser realizadas por el estudiante.

El desarrollo del sistema didáctico sigue una lógica que establece las acciones siguientes:

a) Transformación de los datos de la actividad de estudio de Física con el fin de poner al descubierto la relación universal del objeto estudiado;

b) Modelación de la relación diferenciada en forma objetal, gráfica o por medio de símbolos;

c) Transformación del modelo de la relación para estudiar sus propiedades en "forma pura";

ch) Control sobre el cumplimento de las acciones anteriores;

d) Evaluación de la asimilación del procedimiento general como resultado de la solución de la actividad de estudio de Física presentada.

Cada una de estas acciones está compuesta de las correspondientes operaciones, cuyo conjunto cambia según las condiciones concretas en que se resuelve una u otra actividad de estudio [Davidov, 1988, pg 181].

\section{Evaluación en el proceso de enseñanza- aprendizaje del sistema didáctico propuesto}

El aprendizaje de la Física supone que además de la adquisición de conocimientos relativos al contenido de la ciencia Física, el estudiante tiene que desarrollar 
su capacidad de generalización, reflexión, pensamiento independiente y crítico necesarios a su especialización.

Para valorar la calidad del aprendizaje logrado se efectuaron mediciones no solo de los aspectos que tradicionalmente se exigen, sino también indicadores cualitativos como el grado de generalización, de reflexión o conciencia, de independencia, dominio y solidez del conocimiento [González, 1995, pg 7].

Para Vygotsky, la evaluación que sólo enfoque el nivel real de rendimiento o desarrollo de un individuo es incompleta, o proporciona un cuadro parcial. Para obtener una evaluación completa es necesario evaluar al individuo en el segundo nivel, es decir, en la zona de desarrollo próximo.

[Campione, 1989, pg 151-165] creó una taxonomía útil de los diferentes enfoques de la evaluación dinámica; estas diferencias pueden entenderse con respecto a las posiciones que ocupan en tres dimensiones: foco, interacción y objetivo. El Foco, se refiere a las diferentes formas por las cuales el potencial de cambio está evaluándose; esto se da por la búsqueda de un número mayor de puntos, usándose un procedimiento prueba-enseñanza-prueba, o por la observación de los procesos subyacentes implicados en el aprendizaje o en el cambio.

En el proceso de enseñanza-aprendizaje de la Física y en particular para los estudiantes del primer año de los cursos de las áreas tecnológicas, las dificultades en el aprendizaje de la Física se acentúan, pues se supone que éstos presenten una estructura cognoscitiva adaptada a la adquisición de nuevos conocimientos y habilidades y de esa manera pasen a un nivel qualitativamente superior.

En esta perspectiva, al enseñar a los estudiantes nuevos conocimientos y habilidades, estamos modificando su estructura cognoscitiva, es decir, estamos modificando los conocimientos y habilidades, así como operando una transformación y formación de una nueva estructura cognoscitiva que recoge las características necesarias para desarrollar el aprendizaje al nivel que se desea.

En el proceso de enseñanza-aprendizaje de la Física podemos utilizar por ejemplo la Actividad de Estudio de Física e Tareas Diagnósticas para evaluar el potencial del cambio del estudiante.

La Interacción, se refiere al tipo de relación entre el estudiante y el profesor; puede decirse que es parte de un continuo, con un lado estructurado y estandarizado y otro más desestruturado y clínico.

En el proceso de enseñanza-aprendizaje de la Física, tenemos la relación del profesor (mediador) con el alumno (mediado) donde el profesor observa las actitudes de los estudiantes, así como usa todas las respuestas de pruebas formales (los puntos fuertes y los puntos débiles), para la retroalimentación del proceso que busca una mejora del potencial del cambio del estudiante.
El Objetivo, se refiere al tipo de habilidades que están teniéndose en cuenta en la evaluación; ésas pueden ser las habilidades específicas y procesos relacionados con el plan de estudios o con áreas de dominio específico, o habilidades más generales y globales.

Al construir el conocimiento de la ciencia Física, el estudiante tiene que adquirir ciertas habilidades asociadas a su especialización, como por ejemplo, identificar, generalizar, aplicar, la habilidad de la modelación y experimentación de un objeto de estudio ligado a un fenómeno físico, es decir, para encontrar la solución del problema, el estudiante tiene que hacer algunas acciones necesarias para hacer efectiva la actividad de estudio de la Física, como: transformar el objeto de estudio, desarrollar el modelaje a través de la representación matemática y realizar la experimentación del modelo matemático del fenómeno estudiado en equipos disponibles en los laboratorios, o usar la simulación numérica del fenómeno a través de la computadora.

En este contexto, el estudiante adquiere habilidades de solución de problemas teóricos y usa la demostración experimental en clase, tornándose activo, participativo, así como, objeto y sujeto de la transformación.

En esta dimensión el profesor puede hacer estudios cualitativos para saber qué tipo de ayuda el estudiante necesita para desarrollar su potencial de cambio.

En síntesis, "no es suficiente exponer un individuo a un conjunto de informaciones o contenidos (por ejemplo, cursos de formación) por más rico que éste sea; es necesario establecer una didáctica interactiva entre el mediador y el mediado que permita la expansión del máximo de la capacidad de pensamiento" [Fonseca, 1998, pg. 261].

Al realizar la actividad de estudio de la Física como parte del proceso de enseñanza- aprendizaje de la Física, el estudiante desarrolla las estrategias de aprendizaje necesarias para realizar tal actividad.

\section{Diseño metodológico}

Para Vygotsky, el aprendizaje es una actividad social y no sólo un proceso de realización individual, como lo pensaron hasta ahora; una actividad de producción y reproducción del conocimiento por medio de la cual el individuo asimila los modos sociales de actividad y de integración, y después en la escuela, asimila los fundamentos del conocimiento científico, bajo las condiciones de orientación y de interacción social.

El curso siempre se desarrolla creando una atmósfera favorable a la adquisición de conocimientos y habilidades. Los estudiantes reciben orientación teórica necesaria para la comprensión de los fenómenos físicos analizados experimentalmente. El contenido sigue una organización lógico sistémica estructural-funcional, a partir de las características esenciales más genéricas, buscando crear en el estudiante las condiciones cognos- 
citivas para que éste pueda desarrollar el proceso de absorción de conocimiento y habilidades, de acuerdo con los objetivos presentados.

La metodología a ser empleada se apoya en las acciones siguientes:

a) La transformación de las condiciones del problema, con vistas a descubrir sus relaciones esenciales, entre aquéllas que se extrajeron de las características del objeto estudiado;

b) La creación de un modelo de las relaciones tratadas precedentemente, bajo la forma material, gráfica, semántica, o de generalizaciones simbólicas;

c) La experimentación como transformación del modelo, con vistas a estudiar las propiedades intrínsecas a estas relaciones;

ch) La creación de un sistema de problemas específicos, que pueden resolverse por medio de la aplicación de la manera general;

d) El control de la realización de las acciones precedentes;

e) La evaluación de la adquisición de la manera general como resultado de la resolución de un problema de aprendizaje.

La metodología utiliza Actividades de Estudio de Física (Anexo I), y éstas son formadas por un grupo de acciones, cada una de las cuales con su propio problema de aprendizaje a ser resuelto, durante el proceso de enseñanza-aprendizaje de una asignatura de Física.

\section{Muestra}

La muestra fue compuesta de estudiantes de la asignatura Física Teórica y Experimental I de los cursos de Ingeniería. En estas especialidades, la Disciplina Física General se divide en cuatro asignaturas: Física Teórica y Experimental I, II, III y IV. Nuestro experimento tuvo lugar a través de la asignatura Física Teórica y Experimental I, cuyo contenido programático fue reestructurado (Física General I) para ayudar a la propuesta de la investigación, con modificaciones en los objetivos, los contenidos y en el proceso de enseñanza-aprendizaje.

\section{Análisis crítico de los resulta- dos de la investigación}

El proceso de enseñanza-aprendizaje de la Física se ejecuta en la base de la asimilación por parte de los estudiantes del contenido de la asignatura Física. El eje de la asignatura es su programa, así como la descripción sistemática y jerárquica del conocimiento y habilidades que deben ser asimilados por los estudiantes.

El programa fija el contenido de la asignatura, y, a su vez, los métodos de enseñanza-aprendizaje definen el carácter del material didáctico, de la planificación de la enseñanza, los controles, evaluaciones, etc.

El perfeccionamiento del proceso enseñanzaaprendizaje de la Física a partir de que sus contenidos han sido seleccionados de la ciencia Física y estructurados en función del invariantes, lo que contribuyen al desarrollo del modo de actuación del estudiante en su especialización desde una asignatura básica.

La relación entre el objeto de estudio de la asignatura Física General I y el método de enseñanzaaprendizaje ( atividad de estudio), modo de actuación de profesores y estudiantes, es lo que da origen a la secuencia de los contenidos - sistemas de conocimientos y sistemas de habilidades de la asignatura Física General I, y que permite el aprendizaje a partir del modo de actuación del estudiante.

El método de alcanzar lo concreto a partir de lo abstracto es una forma que permite al pensamiento resolver el problema del conocimiento científico de la realidad; esto implica en un método que permite transformar en conceptos los datos de la intuición y de la representación de la realidad del mundo.

En este proceso de adquisición del conocimiento es necesaria la evaluación crítica de los conceptos anteriores; esta evaluación no puede realizarse sin el análisis de los hechos empíricos, y, de la misma manera, el análisis teórico de tales hechos es imposible de ser cumplido si no se tiene los conceptos a través de los cuales pueden expresarse tales hechos.

El razonamiento deductivo es uno de los momentos fundamentales del proceso de adquisición del conocimiento humano.

De acuerdo con Hernández, concebimos la deducción como un procedimiento que permite el desarrollo real de los conocimientos, apoyado en la diversidad de hechos en su movimiento e interrelación interna que incluye en ella el momento empírico, porque contiene el análisis más riguroso de los hechos empíricos a través de la inducción. Esta concepción permite entender y reproducirse el desmembramiento interior del objeto en el movimiento de los conceptos y con él partir del análisis de las formas más simples del desarrollo del todo investigado, al análisis de las formas más complejas, genéticamente secundarias. La deducción se aproxima, de verdad, a la comprensión teórica de los hechos empíricos [Hernández, 1992, pg 33].

\section{Tareas diagnósticas}

Las "Tareas Diagnósticas" no sólo se proponen para informar las posibilidades actuales del individuo, sino también para medir su potencialidad de desarrollo. Es necesario partir de la consideración de que las capacidades humanas se forman y se desarrollan a lo largo de la vida y el papel de la educación es predominante en este proceso; para alcanzar tal propósito, las Tareas presentadas intentan no sólo verificar el grado de adecuación de las respuestas, sino también el proceso de formación de las acciones mentales u operaciones lógicas de Galperin. 
Tal teoría nos permite, además, satisfacer nuestro interés de caracterizar las propias acciones de la deducción, no sólo desde el punto de vista cuantitativo, sino en su desarrollo cualitativo a través del estudio de los parámetros o características de la acción. De esta manera, es posible profundizar en su esencia y dirigir nuestros pasos conscientemente para mejorar la formación de estas habilidades en el estudiante.

Las Tareas Diagnósticas pretenden medir algunas propiedades de las acciones lógicas, objeto de estudio, que a su tiempo, servirán como base para evaluar el nivel de desarrollo del procedimiento de deducción.

Las propiedades de la acción que se estudiaron y que conducen al desarrollo del estudiante con vistas a su especialidad técnico-científica, son [Hernández, 1992, pg 39]:

a) el grado de generalización de la acción que es determinado por la extensión del concepto o el límite de aplicación de la acción a partir de las cualidades esenciales del mismo; eso permite al individuo hacer generalizaciones adecuadas en el análisis de un caso particular. La generalización esencial es basada en los marcos de referencia de la orientación, es por esto que es necesario trabajar con una base orientadora completa y generalizada que requiere una organización sistémica estructural-funcional del contenido del objeto de estudio y el uso de las situaciones típicas y lógicas.

b) el grado de conciencia, muy relacionado con la forma verbal, según la teoría de las etapas de formación de acciones mentales de Galperin, consiste en la posibilidad que tiene el individuo, no sólo de ejecutar correctamente la acción, sino de fundamentar en forma verbal su ejecución correcta, demostrando un grado de reflexión sobre lo que hace, que le permite explicar la lógica de su acción.

Dependiendo de los elementos usados por el individuo para justificar su afirmación, es posible medir cuánto él está consciente o no de las propiedades esenciales que le permiten tomar una o otra decisión.

c) el grado de independencia que se refiere a las posibilidades que tiene el individuo de ejecutar una acción correctamente de una manera independiente $o$ con algún nivel de ayuda. No siempre es posible realizar una nueva acción, desde el principio, de una manera independiente; de esta forma, en su proceso de la formación, es necesario planificar niveles de ayuda (mediación) para garantizar la realización correcta de la acción en el propio proceso de enseñanza-aprendizaje de la Física, ya que no hay un dominio del mismo.

El contenido de las Tareas usadas comprenderá dos áreas: Vida Cotidiana y Física (Anexo II). Estos dos tipos de contenido buscan comprender de una manera global la actividad cognoscitiva del hombre, tanto respecto al conocimiento empírico, como al conocimiento teórico.

El conocimiento empírico cotidiano, es propio de todos los hombres, porque se adquiere en la práctica social humana para la cual no es necesaria una preparación especial ya que está históricamente muy unido a la experiencia individual y a las tradiciones históricamente formadas; aquí el objetivo fundamental del acto cognoscitivo es la solución de problemas y dificultades con que se enfrenta el hombre en su actividad cognoscitiva.

Por otro lado, el conocimiento teórico expresa un conocimiento que no se da directamente al hombre. Este tipo de conocimiento puede alcanzarse con una preparación especial para él, proceso este que tiene como propio fin la obtención de conocimientos, para lo cual necesita medios especiales de conocimiento que le permitan penetrar en los objetos de la naturaleza, describirlos, modificar su conducta y demostrar su esencia y su característica que reflejen las leyes que gobiernan el desarrollo.

\section{Análisis crítico y descripción de los resultados de la investigación}

\section{Características de la Muestra}

La muestra fue compuesta por dos grupos de estudiantes de la Asignatura Física Teórica y Experimental I de los cursos de Ingeniería. El Grupo Experimental tería 39 estudiantes del Curso de Ingeniería Química. El Grupo de Control, tería 54 estudiantes del Curso básico de las especialidades de Ingeniería Civil, Mecánica y Eléctrica.

2. Análisis de los resultados de los Instrumentos Grupo de Tareas 1 (GT1), Grupo de Tareas 2 (GT2) y Grupo de Tareas 3 (GT3).

Los Instrumentos Grupos de Tareas 1, 2 y 3, tienen como propósito medir los grados de Conciencia, Generalización e Independencia, alcanzados por los estudiantes debido a los métodos de enseñanza usados, tanto en el Grupo de Control (Enseñanza Tradicional), como en el Grupo Experimental, que usa el nuevo método de enseñanza-aprendizaje de la Física para el área tecnológica.

Los criterios de cuantificación de las respuestas son:

a) BF - Bien Fundamentada; RF - Regularmente Fundamentada; MF - Mal Fundamentada y SF - Sin Fundamentación.

b) Muy Alto - + 85\% de aciertos; Alto - de 65 a $85 \%$ de aciertos; Regular - de 50 a $65 \%$ de aciertos y Bajo - menos de 50\% de aciertos [Hernández, 1992, pg 52].

A continuación se describen las Tablas resultados, con sus respectivas análisis:

2.1 Comparación entre los diagnósticos Inicial y Final con relación al grado de Conciencia (en\%) 
Tabla 1 - Comparación entre los diagnósticos Inicial y Final en relación con el grado de Conciencia - en (\%)

\subsection{Grupo Experimental}

\begin{tabular}{|l||c|c|}
\hline Grado de Conciencia & Inicial & Final \\
\hline \hline Resp. Correctas & 76,28 & 81,84 \\
\hline \hline BF & 59,19 & 80,13 \\
\hline RF & 8,33 & 0,42 \\
\hline MF & 6,20 & 0,85 \\
\hline SF & 2,56 & 0,44 \\
\hline \hline Resp. Incorrectas & 23,72 & 18,16 \\
\hline \hline BF & 4,06 & 5,55 \\
\hline MF & 16,88 & 11,97 \\
\hline SF & 2,78 & 0,64 \\
\hline
\end{tabular}

1.2 Grupo de Control

\begin{tabular}{|l||c|c|}
\hline Grado de Conciencia & Inicial & Final \\
\hline Resp. Correctas & 73,76 & 55,87 \\
\hline \hline BF & 48,30 & 41,66 \\
\hline RF & 14,20 & 3,41 \\
\hline MF & 6,64 & 3,98 \\
\hline SF & 4,62 & 6,82 \\
\hline \hline Resp. Incorrectas & 26,23 & 44,13 \\
\hline \hline BF & 3,86 & 0,95 \\
\hline MF & 15,12 & 31,26 \\
\hline SF & 7,25 & 11,92 \\
\hline
\end{tabular}

Considerando el contenido de las Tablas 1.1 y 1.2 , verificamos, que, al principio de los trabajos, las diferencias entre el Grupo Experimental y de Control eran pequeñas, en que respecta al grado de Conciencia.

Por ejemplo, veamos el número de respuestas correctas bien fundamentadas: el Grupo Experimental obtuvo 59,19\%, mientras que el Grupo de Control tenía $48,30 \%$.

Tabla 2 - Comparación del diagnóstico Final de los Grupos Experimental y de Control (en \%)

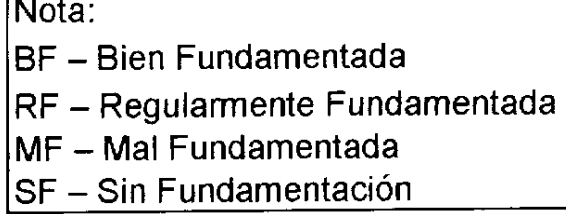

\begin{tabular}{|l||c|c|}
\hline Calidad de las Respuestas & G. Exp. & G. Cont. \\
\hline \hline Resp. Correctas & 81,84 & 55,87 \\
\hline \hline BF & 80,13 & 41,66 \\
\hline RF & 0,42 & 3,41 \\
\hline MF & 0,85 & 3,98 \\
\hline SF & 0,44 & 6,82 \\
\hline \hline Resp. Incorrectas & 18,16 & 44,13 \\
\hline \hline BF & 5,55 & 0,95 \\
\hline MF & 11,97 & 31,26 \\
\hline SF & 0,64 & 11,92 \\
\hline
\end{tabular}

Nota:
BF - Bien Fundamentada
RF - Regularmente Fundamentada
MF - Mal Fundamentada
SF - Sin Fundamentación


La Tabla 2, que representa el resultado Final en términos de Calidad de las Respuestas, viene ser, realmente, un complemento al item anterior (2.1).

Como se observa, los resultados Iniciales de los dos grupos están próximos, como era de esperar; el gran cambio cualitativo ocurre en el resultado final, en que hay una evolución considerable del Grupo Experimental, en relación con el Grupo de Control.

Como se ve en la Tabla 2, el total de respuestas correctas del Grupo Experimental es 81,84\%, contra
$18,16 \%$ incorrectas. Por otro lado, en el Grupo de Control tenemos $55,87 \%$ correctas, contra $44,13 \%$ incorrectas.

Esto demuestra una mayor preparación del Grupo Experimental en realizar deducciones y fundamentarlas, usando todas las características esenciales necesarias.

2.3 Comparación entre los diagnósticos Inicial y Final en relación con el Grado de Generalización (en\%)

Tabla 3 - Comparación entre los diagnósticos Inicial y Final con relación al Grado de la generalización (en\%).

1. Grupo de la

Experiencia

\begin{tabular}{|l||c|c|c|c|c|c|}
\hline \multicolumn{1}{c||}{$\begin{array}{c}\text { Grado de } \\
\text { Generalización }\end{array}$} & \multicolumn{6}{c|}{ Situaciones lógicas } \\
\cline { 2 - 7 } & \multicolumn{2}{|c|}{ Pertenencia } & No Pertenencia & \multicolumn{2}{c|}{ Indeterminada } \\
\cline { 2 - 7 } & Inicial & Final & Inicial & Final & Inicial & Final \\
\hline Muy Alto & 10,26 & 74,36 & 41,03 & 58,97 & 5,13 & 15,38 \\
\hline Alto & 56,41 & 15,38 & 33,33 & 35,90 & 51,28 & 48,72 \\
\hline Regular & 30,77 & 0,20 & 7,69 & 5,13 & 2,56 & 0,00 \\
\hline Bajo & 2,56 & 10,26 & 17,95 & 0,00 & 41,03 & 35,90 \\
\hline
\end{tabular}

\section{Grupo del Control}

\begin{tabular}{|l||c|c|c|c|c|c|}
\hline \multicolumn{1}{|c||}{$\begin{array}{c}\text { Grado de } \\
\text { Generalización }\end{array}$} & \multicolumn{7}{c|}{ Situaciones lógicas } \\
\cline { 2 - 8 } & \multicolumn{2}{|c|}{ Pertenencia } & No Pertenencia & \multicolumn{2}{c|}{ Indeterminada } \\
\cline { 2 - 7 } & Inicial & Final & Inicial & Final & Inicial & Final \\
\hline Muy Alto & 12,96 & 13,64 & 33,33 & 2,27 & 5,56 & 4,55 \\
\hline Alto & 46,30 & 27,27 & 27,78 & 36,36 & 40,74 & 22,73 \\
\hline Regular & 22,22 & 6,82 & 5,56 & 27,27 & 5,56 & 2,27 \\
\hline Bajo & 18,52 & 52,27 & 33,33 & 34,09 & 48,15 & 70,45 \\
\hline
\end{tabular}

El resultado Inicial y Final relativo al Grado de Generalización muestra un cambio bastante claro en el perfil de los dos Grupos - Experimental y de Control.

En el caso del Grupo Experimental, verificamos que más de $50 \%$ de los estudiantes consigue, al final del curso, tratar situaciones de Pertenencia apropiadamente $(74,36 \%)$ y las de No Pertenencia $(58,97 \%)$. En el caso de las situaciones Indeterminadas, es todavía bajo el número de estudiantes que consiguen tratarlas apropiadamente.
Con respecto al Grupo de Control, a la medida en que el curso evolucionó, su capacidad de tratar situaciones lógicas no tenía la misma evolución. Al contrario, menos de $20 \%$ de los estudiantes consiguen tratar las situaciones de Pertenencia apropiadamente $(13,64 \%)$ y No Pertinencia (2,27\%). En el caso de la situación Indeterminada, apenas $4,55 \%$ de los estudiantes consiguen tratarla apropiadamente.

2.4 Comparación entre los diagnósticos Inicial y Final con relación al grado de Independencia - (en\%)

Tabla 4 - Comparación entre los diagnósticos Inicial y Final en relación con el grado de Independencia - (en\%) 1. Grupo Experimental

\begin{tabular}{|l||c|c|}
\hline $\begin{array}{l}\text { Grado de } \\
\text { Independencia }\end{array}$ & Inicial & Final \\
\hline Independiente & 7,69 & 43,59 \\
\hline Reg. Independiente & 33,33 & 46,15 \\
\hline Poco Independiente & 51,28 & 5,13 \\
\hline Nada Independiente & 7,69 & 5,13 \\
\hline
\end{tabular}

\begin{tabular}{l} 
Nota: \\
Inicial - GT1 \\
Final - GT3 \\
\hline
\end{tabular}


Como puede observarse, inicialmente pocos estudiantes eran capaces de obtener resultados buenos sin ayuda, siendo más que la mitad Poco Independiente $(51,28 \%)$ o Nada Independiente $(7,69 \%)$. Aun así, en el resultado Final, el número de estudiantes que consiguen trabajar de una manera Independiente o Regularmente Independiente es $89,74 \%$. Una vez más se observa la evolución acentuada de los estudiantes del Grupo Experimental, en el qué dice respecto a la Independencia.

\subsubsection{Grupo de Control}

Tabla 5 - Comparación entre los diagnósticos Inicial y Final en relación con el grado de Independencia - (en\%)

2. Grupo de Control

\begin{tabular}{|l||c|c|}
\hline $\begin{array}{l}\text { Grado de } \\
\text { Independencia }\end{array}$ & Inicial & Final \\
\hline \hline Independiente & 0,00 & 2,27 \\
\hline \hline Reg. Independiente & 31,48 & 13,64 \\
\hline Poco Independiente & 29,63 & 31,82 \\
\hline Nada Independiente & 38,89 & 52,27 \\
\hline
\end{tabular}

Los estudiantes del Grupo de Control, inicialmente, son incapaces de realizar su trabajo de deducción lógica de manera totalmente Independiente.

Al final, se encuentra un porciento de $2,27 \%$ qué es capaz de trabajar totalmente de manera Independiente.

Se nota así, que hay, de parte de esos estudiantes, dificultades para identificar todas las características esenciales para su análisis, o los mismos no consiguen analizar apropiadamente las premisas presentadas.

\section{Conclusiones y recomenda- ciones}

La experiencia realizada demuestra que es posible elevar el nivel de desarrollo del pensamiento lógico a través de una Asignatura, como la Física. Los resultados obtenidos en nuestra experiencia demuestran la posibilidad de elevar el desarrollo del procedimiento de deducción cuando esto forma parte de los objetivos de un programa educativo y se estructuran Actividades de Estudio dirigidas con tal propósito.

La estructuración del contenido con el enfoque sistémico estructural-funcional favorece el desarrollo de los procedimientos lógicos del pensamiento, así como, el uso y la organización de las Actividades de Estudio de Física pedagógicamente elaboradas propician la adquisición de conocimiento y habilidades de la asignatura Física.

Las Actividades de Estudio de Física así formuladas superan las dificultades de la enseñanza tradicional y las limitaciones y deficiencias del trabajo experimental que hoy tiene lugar en la enseñanza de la Física y que desvirtúan significativamente la verdadera actividad de la ciencia.

Además, la enseñanza de la Física a través de las Actividades de Estudio de Física demostró que la relación entre la solución de problemas teóricos, en que se usa

\begin{tabular}{l} 
Nota: \\
Inicial - GT1 \\
Final - GT3 \\
\hline
\end{tabular}

la modelación, experimentación y simulación en clase, hace que el estudiante tenga un papel activo en el proceso de enseñanza-aprendizaje de la Física. El desarrollo de ese proceso, a través de las Actividades de Estudio, que consolidan la verificación de la actividad teórica y práctica de la enseñanza de la Física, demostró el desarrollo de habilidades prácticas y teóricas por parte de los estudiantes, haciendo que ellos conciban sus propias ideas con respecto al conocimiento de los conceptos científicos de la Ciencia Física y comprueben su capacidad para resolver problemas a imagen y semejanza de los problemas científicos.

La investigación demuestra que la "ausencia" de un sistema didáctico sistémicamente estructurado de enseñanza-aprendizaje de la Física es una de las razones del fracaso de los estudiantes en el aprendizaje de la Física.

La concepción de este modelo didáctico propuesto, no sólo considera el proceso en símismo, sino también los diferentes momentos por los cuales éste pasa, donde el control y la evaluación cumplen un papel importante, al concebir un grupo de indicadores (conciencia, generalización e independencia), que apuntan a criterios de calidad para la evaluación del aprendizaje del estudiante.

De esa manera, el profesor transfiere la atención del resultado del proceso - aspecto cuantitativo, que evidencia solamente la medición de los resultados en calificaciones finales, al aspecto cualitativo de las etapas en que transcurre el proceso y por las transformaciones que se producen en sus indicadores fundamentales, proporcionando una enseñanza de calidad. Los resultados de la investigación conduzcan a la conclusión de que el modelo didáctico propuesto para la enseñanzaaprendizaje de la Física para el área tecnológica, en particular para las especialidades de Ingeniería, establece un nuevo modelo pedagógico que responde a las exigencias técnico-científicas contemporáneas. 
Para impartir el nuevo modelo de enseñanza, el profesor tendrá que tener habilidad, tanto en el tratamiento con el formalismo matemático, como con la fenomenología del concepto estudiado; es decir, que no exista más en la enseñanza básica de la Física la dicotomía entre la Física teórica y experimental, pasando a tener un único carácter, donde la interrelación de la estructura del concepto físico es uno de los fundamentos de este abordaje. Usar, además de los recursos disponibles en laboratorios de Física, la computadora como medio de enseñanza, para realización de las simulaciones de los fenómenos físicos relacionados en las Actividades de Estudio de Física.

Usar la formulación de las pruebas de evaluación con la siguiente estructura:

- Pregunta teórica de desarrollo conceptual;

- La pregunta de aplicación de SituaciónProblémica;

- Preguntas que usan análisis con argumentación, usando las características esenciales para analizar la deducción.

Considerar el proceso de enseñanza-aprendizaje de la Física de manera sistémica y desarrollado como un conjunto de partes interligadas, y que posee una lógica interna característica, donde el control y la evaluación (cuantitativa y cualitativa) desempeñan un papel importante en la retroalimentación del proceso necesario para conseguir los objetivos planificados, tanto en la visión del profesor, como del estudiante.

A partir de este modelo y de la investigación realizada, es posible establecer un sistema didáctico para la enseñanza de la Física para el área tecnológica, tenendo en cuenta, particularmente, la importancia de la Física en la formación del ingeniero, de manera de contribuir al desarrollo científico-técnico de Brasil.

Pensamos que este trabajo de investigación es una contribución a los estudios para el perfeccionamiento de la educación universitária en Brasil y que a la larga repercutirá en el desarrollo humano, intelectual y educativo del futuro profesional de la Ingenieria.

\section{References}

[1] ALVAREZ, C. M. Z. La Universidad como Instituición Social. Editorial Academia, Habana, 1996.

[2] ARRUDA, J.R.C. Enseñanza-Aprendizaje de la Física para el Área Tecnológica : Un Proceso de Calidad, Tese de Maestría, Habana, 1998.

[3] CAMPione, J. C. Assisted Assessement: A Taxonomy of Aproaches and Outline of Strengths and Weaknesses, Journal of Learning Disabilities 22(3), pg. 151165,1989 .

[4] DAVIDOV, V. Tipos de generalización en la enseñanza. Pueblo Educación, La Habana, 1974.

[5] DAVIDOV, V. La enseñanza Escolar y el Desarrollo Psiquico. Investigación psicológica Teórica y Experimental. Editorial Progresso, Moscu, 1988.
[6] DAVIDOV, V. e Slobódchikov, V. I., La enseñanza que Desarrolla en la escuela del Desarrollo; en La educación y la enseñanza: una mirada al futuro. Ed. Progresso, Moscu, pg 118 - 144, 1991.

[7] FARIÑAS, G. L Maestro: una estrategia para la enseñanza. Editorial Academia, La Habana, 1995.

[8] FONSECA, V. Aprender a Aprender: a educabilidade cognitiva. Porto Alegre: Artmed, 1998.

[9] GALPERIN, P.Ya. Introdución a La Psicología. Editorial Pueblo y Educación, Habana, 1982.

[10] GONZÁleZ, O. P. Inovaciones Didáticas en la Educación Superior. Pedagogia 95. CEPES. Universidad de La Habana, 1995.

[11] HERNÁNDEZ, A. D. Estudio y Desarrollo del Procedimiento de Deducción en Estudiantes de Ciencias Tecnicas. Propuesta de Tareas para su Diagnóstico y Formación. Tesis de Doctorado. Ciudad de La Habana, 1992.

[12] KUHN, T.S. A Estrutura das Revoluções Científicas. Editora Perspectiva, São Paulo, 1998.

[13] LEOnTIEV, A.N. Actividad, Consciencia y Personalidad. Editora Pueblo y Educación. Ciudad de La Habana, 1983.

[14] LÓPEZ, M. L. Cómo enseñar a determinar lo esencial. Pueblo y Educación. Ciudad de La Habana, 1989.

[15] PIAGET, J. e INHELDER, B. Da Lógica da Criança à Lógica do Adolescente. Tradução Dante Moreira Leite. Livraria Pioneira Editora, São Paulo, 1976.

[16] SAlmina, N. G. La Actividad Cognoscitiva de los Alumnos y el Modo de Estucturar la Asignatura. Universidad de La Habana, 1989.

[17] TALÍZINA, N. F. La Formación de la Actividad Cognoscitiva de los Escolares. Universidad de la Habana, La Habana, 1987.

[18] TALÍZINA, N..F. Psicologia de la enseñanza. Editora Progresso, Rusia, 1988.

[19] TALíZINA, N. F. Conferencia Sobre "los fundamentos de la enseñanza en la educación superior" Universidad de la Habana-Cuba, 1997.

[20] VYGOTSKY, L.S. A Formação Social da Mente. Livraria Martins Fontes Editora Ltda - São Paulo, Brasil, 1989.

[21] VYGOTSKY,

L.S. Pensamento e Linguagem. Tradução Jefferson Luiz Camargo. São Paulo, Martins Fontes, 1993.

\section{ANEXO I - Actividades de Estu- dio La Ciencia Física}

La palabra griega Physis quiere decir naturaleza; por esta razón llamaro a la Física la ciencia de la naturaleza. El estudio de muchos años de los fenómenos de la naturaleza, llevó a los científicos a la idea de la materialidad del mundo que nos rodea. De esa manera, 
todo lo que realmente existe en la naturaleza es material. Por consiguiente, nuestra idea sobre la naturaleza se basa en la concepción materialista del mundo.

La observación y estudio del mundo que nos rodea, demostró que la materia está en constante movimiento. El movimiento de la materia puede cambiar de forma, pero, este no se crea ni se elimina. Es decir, el mundo que nos rodea es la materia en perpetuo movimiento y desarrollo. La medida general de la materia, en todas sus formas es la energía, y la constancia del movimiento de la materia se expresa en la ciencia por la ley de la conservación de la energía.

Por consiguiente, la Física estudia diferentes formas de movimiento de la materia, sus transformaciones mutuas, así como las propiedades de la substancia y del campo, es decir, la Física tiene como objetivo el estudio de los componentes de la materia y sus respectivas interacciones.

\section{La Asignatura Física}

El abordaje del contenido en el proceso de enseñanza de una ciencia dada, en particular la Física, se constituye en una unidad desde el punto de vista didáctico e ideológico, donde lo que enseñamos es parte de las demandas de la sociedad, y está presente a través de los contenidos impartidos, que contribuyen a la construcción del conocimiento y la adquisición de habilidades y hábitos de los estudiantes, así como posibilitan a éstos: autodeterminación, independencia profesional, una formación técnica y ética, adquiriendo valores de manera de formarse como ciudadano.

El contenido de la enseñanza de la Física debe entenderse como: un sistema de conocimientos, habilidades y hábitos, sobre la naturaleza, la sociedad, el pensamiento, la técnica y los modos de actuación cuya asimilación garantiza la formación en la conciencia del estudiante, de una imagen del mundo, correcta y preparada con un enfoque metodológico adecuado a la actividad cognoscitiva y práctica.

El conocimiento de la ciencia Física se expresa a través de los conceptos físicos y términos fundamentales sin los cuales no se puede comprender ningún texto, así como los conceptos del lenguaje natural que refleje la actividad cotidiana, como a los conceptos científicos; las leyes fundamentales de la ciencia Física que revelan los eslabones y relaciones entre los distintos objetos y fenómenos de la realidad. Las teorías de la Física contienen un sistema de conocimientos científicos sobre un determinado conjunto de objetos y sobre los métodos de explicación y prescripción de los fenómenos físicos.

Estos contenidos de enseñanza de la Física forman parte de las Asignaturas del plan de estudio, de los libros didácticos e informaciones científico-técnicas, que son por consiguiente la base y los fundamentos de la ciencia estudiada o mejor, el contenido de la Asignatura, donde está presente el conjunto (sistema) de conocimientos científicamente elaborados.

\section{Procedimientos para realizar una actividad de estudio de física}

La Actividad de Estudio busca orientar el estudiante para la solución de las acciones de estudio; dentro de esta orientación tenemos las siguientes acciones: transformación, modelación, experimentación, control y evaluación.

Describimos a seguir las acciones necesarias para la realización de una Actividad de Estudio.

\section{Transformación}

La transformación, está dirigida a la investigación y al descubrimiento de la relación real entre las condiciones de la tarea, relación ésta que es la base general y la fuente de las peculiaridades de la tarea dada y de todas las tareas similares.

\section{Modelación}

Estamos de acuerdo con Davidov [1988, pg.133] en que "modelo es un sistema representado mentalmente o realizado materialmente que, reflejando o reproduciendo el objeto de la investigación, es capaz de sustituirlo, de modo que su estudio nos dé una nueva información sobre este objeto", es decir, sólo la información que fija la relación inicial, de las condiciones de la tarea del estudio a resolver.

Así, el modelo representa la realidad, pero no tiene todos sus atributos, sólo aquéllos considerados esenciales.

El modelaje consiste en construir modelos a partir del lenguaje científico para lo concreto, de lo general hacia lo particular.

El modelaje de la actividad de estudio consiste en representar de una forma esquemática el planeamiento de lo escrito en ella, así como las condiciones, datos, interrogativas y solicitudes.

Para comprender la Actividad de Estudio es necesario establecer una forma de aislar su esencia, eliminando los elementos que puedan confundir o impedir su solución. Para ello, realizamos la transformación del objeto de la actividad de estudio.

Entre las características del modelaje de una actividad, consideramos que las más importantes, son las siguientes:

a) Representar de la forma más exacta posible la situación-problema propuesta por la actividad de estudio, utilizando una forma esquemática, ilustración o gráfica;

b) Definir los atributos necesarios y suficientes para la posible solución;

c) Adecuar el contenido del estudio al modelo físico, utilizando el máximo de rigor posible.

Es conveniente explicitar que al convertir al estudiante en "aprendiz de su propio aprendizaje", dándole instrumentos para su propia transformación y enseñándole los mecanismos de procedimiento de 
acción, él se torna capaz de enfrentar con independencia no sólo las actividades de Física, sino también, de cualquier otra Asignatura.

Además, el modelaje es un poderoso instrumento de análisis que consolida la esencia del contenido físico para la experimentación.

\section{Experimentación}

La experimentación es otra parte del proceso de enseñanza-aprendizaje que es utilizado por el profesor para alcanzar los objetivos propuestos en la actividad de estudio.

La experiencia Física requiere, como el modelaje de la actividad, un planeamiento teórico previo y medios que garantizan su ejecución práctica, así como, de un lugar apropiado necesario que facilite la actividad de los profesores y estudiantes.

Para comprender el fenómeno de naturaleza física en la construcción del proceso enseñanza-aprendizaje de la Física, se utiliza su reproducción de forma controlada en un laboratorio.

Describimos a seguir las actividades para que la experimentación se torne un procedimiento metodológico para la conducción de una investigación científica dentro del proceso de enseñanza-aprendizaje de la Física.

1) Concebir la experimentación como una respuesta a determinadas preguntas o como parte de la solución de un problema - la modelación, o como una alternativa para controlar las hipótesis formuladas al tratamiento de un contenido de la Asignatura Física, utilizando un procedimiento de carácter investigativo.

2) Elaborar la idea básica de la experiencia con base en la modelación; en particular, imaginar y ensayar en el plan mental, situaciones posibles que puedan usarse para contrastar las hipótesis formuladas.

3) Detallar el procedimiento a seguir, los materiales, los instrumentos y en general, el montaje que será realizado, evaluando la posibilidad de arribar con ellos a los efectos o resultados previstos; considerar el modo de hacer despreciables los efectos no deseables; pensar el modo concreto en que se realizaron las diversas mediciones y se procesaron los datos.

4) Realizar la experiencia, es decir, montar la instalación diseñada, reproducir el fenómeno previsto, hacer las mediciones.

5) Analizar los resultados a la luz de las hipótesis formuladas, del conjunto de conocimientos disponibles y de los resultados aceptados por la comunidad científica, favoreciendo la revisión del diseño, de las hipótesis o además del problema inicialmente propuesto.

6) Ejemplificar y reflejar las posibles perspectivas abiertas por la experiencia realizada; ahondar en diferentes aspectos la situación física examinada, generalizar y elaborar nuevas preguntas, concebir su traslado a otros contextos y aplicabilidad, tener en cuenta los factores inicialmente considerados, concebir otros posi- bles diseños de la experimentación, procedimientos de medición, tratamiento de datos, etc.

\section{Control}

El control tiene como finalidad asegurar al estudiante la corrección del cumplimiento de todas las demás acciones de estudio.

\section{Evaluación}

La evaluación permite determinar si el estudiante asimiló, así como cuánto asimiló, el procedimiento general de solución de la tarea de estudio dada y de sus múltiples modificaciones.

Así, la organización correcta de la actividad de estudio consiste en que el profesor, apoyándose en la necesidad y en la disposición de los estudiantes de dominar los conocimientos teóricos, sabe presentar en un material, la tarea de estudio que puede resolverse a través de las acciones de estudio correspondientes.

\section{Actividad de Estudio Tipo I}

\begin{tabular}{ll} 
Asignatura: & Curso: \\
\hline Estudiante: & Fecha: \\
\hline Tema: & \\
\hline Clase: & \\
\hline Objetivo: & \\
\hline
\end{tabular}

\section{Orientación}

- Fundamentar el conocimiento físico en el tema del estudio

- Formular la Situación-problema;

\section{ETAPAS:}

\section{ETAPA $n+1$}

Analizar la Actividad de Estudio propuesta, describir la lógica y los factores que son indispensables para la solución del problema propuesto.

ETAPA $n^{0} 2$

Modelar el objeto de estudio: definir las magnitudes y atributos necesarios y suficientes, construir la formulación matemática de la solución del problema.

ETAPA n⿳⺈ 3

En grupo de tres estudiantes y con base en el modelaje de la situación-problema, analice los elementos y propiedades esenciales y las magnitudes físicas implicadas en el fenómeno, así como, la interrelación del concepto físico (modelo, fenomenología y formalismo matemático) definido.

ETAPA n⿳⺈ 4

Elaborar el procedimiento/planeamiento de la experiencia a ser realizada: objetivos, materiales, medios necesarios a la realización del experimento, tipos de control y modos de obtención y tratamiento de los datos. 
ETAPA n 95

Realizar el experimento: mantener el esquema planeado, reproducirse el fenómeno previsto y hacer las observaciones, las mediciones y registrar datos.

I) Valores, Cálculos y Gráficos

II) Preguntas

ETAPA n? 6

En grupo de tres estudiantes, analizar los resultados a la luz de las hipótesis formuladas, del conjunto de conocimientos disponibles y de los resultados de otros investigadores (grupos de estudiantes, resultados aceptados por la comunidad científica), favoreciendo la revisión del diseño de la experiencia, de las hipótesis y además del problema inicialmente formulado.

ETAPA n? 7

Elaborar individualmente un informe que sintetice la actividad (acciones fundamentales) realizada, resaltando los aspectos de interés más grande y relevancia como resultado de la experimentación, para ser presentado y discutido al final de la Actividad de Estudio. ETAPA $n \div 8$

Ejemplificar y reflejar sobre las posibles perspectivas abiertas por el experimento realizado, profundizar en diferentes aspectos la situación física examinada, generalizar y elaborar nuevas preguntas, concebir su transporte para otros contextos y aplicabilidad, tener en cuenta los factores inicialmente no considerados, concebir otros posibles diseños de la experimentación, etc.,

\section{Actividad de Estudio Tipo II}

\begin{tabular}{ll} 
Asignatura: & Curso: \\
\hline Estudiante: & Fecha: \\
\hline Tema: & \\
\hline Clase: & \\
\hline Objetivo: & \\
\hline
\end{tabular}

\section{Orientación:}

Demostrar un fenómeno físico y definir el concepto físico a partir de los elementos esenciales y de la interrelación entre el modelo, formalismo matemático y fenomenología del concepto considerado.

\section{ETAPAS}

1. Concebir la realización de la Actividad de Estudio, como respuesta a ciertas preguntas o como parte de la solución de un problema.

2. Identificar las magnitudes, propiedades esenciales y parámetros del fenómeno observado.

3. Construir el concepto del fenómeno observado. Definir los elementos esenciales.

4. Formular una situación-problema del objeto estudiado.

5. Analizar semánticamente la situación-problema. Describir el procedimiento lógico y los factores que son indispensables para la solución del problema - la transformación del objeto de estudio.
6. Modelar el objeto del estudio: Definir la magnitud, parámetros y atributos necesarios y suficientes, elaborar la formulación matemática de la solución del problema.

7. Experimentar o simular la formulación matemática de la modelación de la solución del problema.

8. Elaborar el esquema lógico de los conceptos implicados en la actividad de estudio.

9. Ejemplificar y reflejar el concepto estudiado, profundizar en diferentes aspectos la situación física examinada, generalizar y elaborar nuevas preguntas, concebir su translación a otros contextos y aplicabilidad.

\section{Actividad de Estudio Tipo III}

\begin{tabular}{ll} 
Asignatura: & Curso: \\
\hline Estudiante: & Fecha: \\
\hline
\end{tabular}

\section{Objetivo}

Realizar una investigación de carácter científico como parte del proceso de enseñanza-aprendizaje de la Física, a partir del contenido de la Asignatura Física General I (Mecánica) y elaborar una investigación de manera de usar el método científico en la solución del problema estudiado y que esto esté en conexión con su especialidad.

El desarrollo de la investigación tiene como base los siguientes ítems:

a) Investigación bibliográfica;

b) Elaboración teórica;

c) Formulación de hipótesis;

ch) Modelación;

d) Experimentación;

e) Resultados y conclusiones.

Asunto investigado:

Resumen:

\section{ANEXO II - Instrumentos de eval- uación}

Las Tareas Diagnósticas siguen la orientación de las experiencias de J. Piaget [Piaget, 1976], así como de las investigaciones de P. Ya. Galperin [Galperin, 1982], apoyadas en la teoría de Vygotsky [Vygotsky, 1989] e [Vygotsky, 1993] de "la zona de desarrollo próximo." 
El contenido de las Tareas utilizadas engloba dos áreas: la vida cotidiana o contenidos abstractos y contenidos específicos más ligados a la ciencia Física. Estos dos tipos de contenido responden a la distinción que se hace en la actividad cognoscitiva del hombre y del conocimiento empírico y el conocimiento teórico (científico) y, aunque los dos estén dirigidos al estudio de objetos reales que se convierten en objeto del conocimiento, en el modo de separación de estos objetos y en la metodología de su inserción en el proceso de conocimiento, se presentan importantes diferencias que es necesario conocer para el estudio del pensamiento humano. En todos los grupos de tareas existen tareas de dos tipos: un primer tipo de tareas en el que el sujeto sólo tiene que evaluar la certeza de la deducción expuesta y fundamentar su evaluación. En este caso, el estudiante, a partir de un análisis de las premisas, debe evaluar si la conclusión hecha está basada en los elementos esenciales necesarios, lo que reflejará en la argumentación de su respuesta.

Un segundo tipo de tareas, más complejas, exige más de la actividad intelectual del estudiante; en este caso, el sujeto tiene que arribar de modo independiente a la deducción a partir de las premisas (que pueden ser verdaderas o probables), y argumentar su conclusión, lo que exige una reflexión sobre los elementos esenciales expuestos en las premisas.

El diseño de estos tipos de tareas permite valorar la calidad de las respuestas cuando la solución tiene sólo un carácter intuitivo, es decir, es parte del reconocimiento o identificación de lo ya dado para el análisis de la veracidad de la conclusión; y cuando son de carácter reflexivo dado por el análisis consciente del sujeto sobre aquellos componentes de las premisas que son esenciales para derivar el nuevo conocimiento o las nuevas relaciones entre los conocimientos que expresan la manifestación particular del fenómeno que se analiza.

En ambos tipos de tareas se puede argumentar, lo que implica la abstracción de las características esenciales, para el tratamiento del objeto concreto, y con él indujimos al estudiante a fijar estas características en palabras que adquieren para él un significado, es decir, ello hace consciente el proceso de conversión de la lógica de las acciones en lógica de los conceptos.

\section{Grupo de Tareas 1}

Nombre: Fecha:

Este grupo de tareas es parte del estudio sobre el desarrollo del pensamiento científico en los estudiantes de nivel superior.

Observe que son dos tipos de tareas. En la primera, es necesario valorar si están correctas o no las conclusiones y el por qué de su respuesta; en la segunda, es necesario elaborar individualmente las conclusiones, teniendo en cuenta las premisas dadas.

Reflexione siempre antes de contestar, procure prestar la mayor atención posible.

\section{Tareas Tipo I}

Valore si las conclusiones a que se arriba se deducen realmente de las premisas que le anteceden. Fundamente su respuesta.

\section{Parte A - Vida Cotidiana}

1. Si un estudiante es académicamente brillante, él tiene promedio 5. Este estudiante tiene promedio 3.

Luego este estudiante no es académicamente brillante.

La conclusión es correcta o incorrecta.

Fundamente

2. Se tiene 4 patas y come hierba es un animal.

Esto tiene 4 patas y come hierba.

Luego, esto no es un animal.

La conclusión es correcta o incorrecta.

Fundamente

3. Si un hombre tiene fiebre está enfermo.

Este hombre está enfermo.

Luego, este hombre tiene fiebre 
La conclusión es correcta o incorrecta.

Fundamente

\section{Parte B - Física}

5. Consideramos el caso del movimiento rectilíneo con aceleración constante. Supongamos que el intervalo $\Delta t$ sea medido a partir del instante en que las manecillas del reloj marcan cero.

Entonces:

$\Delta t=t_{2}-t_{1}=t_{2}-0=t$.

En el instante $t=0, v_{1}=v_{0}$; en el instante $t, v_{2}=v_{0}$.

Por consiguiente, $a(t)=\frac{v-v_{0}}{t} 0: v_{0}+a t$.

Así siendo, los gráficos de este tipo de movimiento pueden ser:
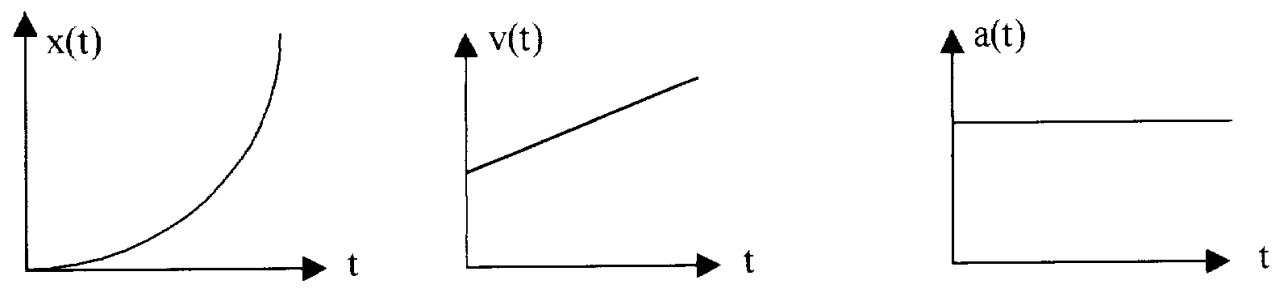

La conclusión es correcta o incorrecta:

Fundamente

6. Cualquier cuerpo lanzado hacia arriba (o abajo) sufre una aceleración igual a la de cualquier cuerpo que cae a partir del reposo. Una vez en caída libre, todos los cuerpos tendrán una aceleración hacia abajo, igual a la aceleración de la gravedad. Un niño, en el tope de un edificio, toca una pelota hacia abajo.

Despreciando la resistencia del aire, y considerando que la pelota sale del reposo y el punto de partida está en $t_{0}=0$, entonces, para $t=1 \mathrm{~s}$, la velocidad es $v=-9,8 \mathrm{~m} / \mathrm{s}$ y la aceleración es $a=-4,90 \mathrm{~m}$.

La respuesta es correcta o incorrecta:

Argumente:

7. La tercera ley de Newton afirma que, cuando hay interacción de 2 cuerpos, la fuerza que el cuerpo 2 ejerce sobre el cuerpo 1 es la misma y opuesta que la fuerza que el cuerpo 1 ejerce en el cuerpo 2 .

Consideremos el caso de un cuerpo en reposo en una mesa. Podemos afirmar que la fuerza normal es igual a la fuerza que el cuerpo ejerce sobre la mesa.

La conclusión es correcta o incorrecta:

Fundamente:

\section{Tareas Tipo II}

¿. A que conclusión se puede arribar a partir de las afirmaciones siguientes? Fundamente su respuesta.

\section{Parte A - Vida Cotidiana}

1. Si un individuo se lavó la cabeza tiene el pelo mojado.

Este individuo se lavó la cabeza.

Luego

Fundamente_

2. Si esta sustancia es aceite no se disuelve en agua.

Esta substancia no es aceite. 
Luego

Fundamente

3. Si una figura geométrica tiene 3 líneas que se cortan formando tres ángulos es un triángulo.

Esta figura tiene 4 líneas que se cortan formando tres ángulos.

Luego

Fundamente

\section{Parte B - Física}

4. Un cuerpo mantiene su estado de reposo o movimiento rectilíneo uniforme si se cumple que:

La acción de otros cuerpos sobre él es cero o la acción resultante es cero.

Cuando un paracaidista abre el paracaída, la acción de la Tierra y del aire sobre él, transcurrido cierto tiempo, se compensan, anulándose el valor de la acción externa sobre él.

Podemos concluir entonces que:

Argumente

5. Un cuerpo conserva su estado de reposo o movimiento rectilíneo uniforme mientras la acción de otros cuerpos no lo obliguen a salir de dicho estado. Esto constituye el enunciado de la primera ley de Newton.

Consideremos un cuerpo en movimiento, las fuerzas que actúan sobre él, y la relación entre los valores de estas fuerzas:

$\mathrm{F}$ aplicada $=\mathrm{F}$ de fricción;

$\mathrm{F}$ normal $=\mathrm{F}$ de la gravedad

Luego, podemos concluir que:

Argumente

6. Si sobre un cuerpo la acción externa resultante es cero, el cuerpo mantiene su estado de reposo o de movimiento rectilíneo uniforme. Una esfera pequeña se dejó caer en la superficie de un líquido y comienza a bajar aceleradamente debido a la acción de la fuerza gravitatoria hacia abajo y la fuerza de fricción viscosa hacia arriba; si transcurrido un tiempo estas fuerzas sobre la esfera se compensan.

Podemos concluir que:

Argumente

\section{Grupo de Tareas 3}

Nombre: Fecha:

En la continuación de nuestro trabajo, presentamos dos tipos de tareas: uno en que es necesario valorar si se adapta o no la deducción arribada y fundamentar la conclusión obtenida; otro en que es necesario, de una forma independiente, inferir la deducción a partir de las premisas dadas en cada caso y argumentar la conclusión obtenida.

Es necesario tener en cuenta que la consecuencia de las premisas es una proposición que no puede ser falsa si las premisas son verdaderas; las premisas pueden ser afirmativas (de la afirmación de una base a la afirmación de una consecuencia) o negativas (de la negación de una base a la negación de la consecuencia).

Éstos conocimientos pueden proporcionar conclusiones tanto verdaderas como probables, por consiguiente, es primeramente necesario verificar si las premisas presentadas son verdaderas o falsas.

Para realizar esta operación, analice se la afirmación colocada en la primera premisa se basa en las características esenciales del concepto que se relaciona en tal juicio. 


\section{Tareas Tipo I}

\section{Parte I - Vida Cotidiana}

Valore si las conclusiones a que se arriba se deducen realmente de las premisas que le anteceden. Fundamente su respuesta.

1. Si un trabajador es ejemplar, ejecuta sus obligaciones.

Luís es trabajador ejemplar.

Luego, Luís no ejecuta sus obligaciones.

La conclusión es correcta o incorrecta.

Fundamente

2. Si un deportista es campeón mundial, es considerado de alto rendimiento deportivo. Juan no es considerado de alto rendimiento deportivo.

Luego, Juan no es campeón mundial.

La conclusión es correcta o incorrecta

Fundamente.

3. Se llueve habrá charcos en la calle.

En estas calles hay charcos.

Luego, llovió en estas calles.

La conclusión es correcta o incorrecta.

Fundamente.

\section{Parte B - Física}

4. Un cuerpo de un sistema mecánico describe oscilaciones libres si en el sistema están presentes las características esenciales de este tipo de movimiento $(\mathrm{a}, \mathrm{b})$.

a) Siempre que el cuerpo se separe de su posición de equilibrio en el sistema surge una fuerza dirigida a dicha posición, la cual obliga el cuerpo a dirigirse nuevamente hacia esta.

b) La fricción en el sistema es despreciable.

Una regla delgada de un metro de longitud esta suspendida por uno de sus extremos, se separa de la posición de equilibrio en un ángulo pequeño y se suelta. Los efectos de la fricción son despreciables. En estas condiciones describe oscilaciones libres.

La conclusión es correcta o incorrecta.

Argumente

5. Un cuerpo de un sistema mecánico realiza oscilaciones amortiguadas si en el sistema están presentes las características esenciales de este tipo de movimiento (a, b).

a) Siempre que el cuerpo se separe de su posición de equilibrio, en el sistema surge una fuerza dirigida hacia dicha posición, la cual obliga al cuerpo a dirigirse nuevamente hacia esta.

b) la fuerza de fricción en el sistema no es despreciable.

Un sistema cuerpo-resorte consiste en un resorte fijo por un extremo y un cuerpo unido al otro extremo capaz de desplazarse, el sistema está sobre una mesa horizontal. El cuerpo se separa de la posición de equilibrio y se suelta. Entre el cuerpo y la mesa y el cuerpo y el aire no existe valor importante de fricción.

Este cuerpo realiza oscilaciones amortiguadas.

La conclusión es correcta o incorrecta.

Argumente:

6. Un cuerpo de un sistema mecánico realiza oscilaciones forzadas si en el sistema están presentes las características (a, b y c) esenciales en este tipo de movimiento.
a) El sistema es capaz de oscilar. 
b) La fricción en el sistema no es despreciable.

c) Sobre el sistema actúa una fuerza periódica exterior.

Se tiene un péndulo simple oscilando y se le aplicó una fuerza.

$\mathrm{El}$ sistema realiza entonces oscilaciones forzadas.

La conclusión es correcta o incorrecta

Argumente

\section{Tareas Tipo II}

\section{Parte I - Vida Cotidiana}

1. Si una mujer tiene hijos es madre.

Júlia es madre.

Luego

Fundamente

2. Si un joven sabe varios idiomas, es políglota.

Helena sabe un idioma.

Luego

Fundamente

3. Si un hombre practica deportes sistemáticamente, será saludable. Este hombre es saludable.

Logo

Fundamente

\section{Parte B - Física}

4. Un cuerpo conserva su estado de reposo o movimiento rectilíneo uniforme mientras la acción de otros cuerpos no lo obliguen a salir de dicho estado. Lo cual constituye el enunciado de la primera Ley de Newton.

Consideremos un cuerpo en movimiento; las fuerzas que actúan sobre él, y la relación entre los valores de estas fuerzas son:

$\mathrm{F}$ aplicada $=\mathrm{F}$ de fricción $\mathrm{F}$ normal $=\mathrm{F}$ gravitatoria.

Luego, podemos concluir que:

Argumente

5. Un cuerpo de un sistema mecánico realiza oscilaciones forzadas, si es capaz de oscilar amortiguadamente y se le aplica una fuerza periódica.

Las fuerzas que actúan sobre el sistema cuerpo-resorte son:

Fuerza elástica;

Fuerza normal;

Fuerza gravitatoria;

Fuerza de fricción;

Fuerza aplicada constante.

El cuerpo realiza oscilaciones forzadas.

La conclusión es correcta o incorrecta:

Argumente. 
6. Si en un sistema mecánico las oscilaciones se mantienen bajo la acción de una fuerza periódica exterior al mismo, se denominan oscilaciones forzadas.

Un niño se mece oscilando en una hamaca y su movimiento tiende a disminuir. Entonces se aplica una fuerza sobre él.

El niño realiza oscilaciones forzadas.

La conclusión es correcta o incorrecta.

Argumente 